University of Nebraska - Lincoln

DigitalCommons@University of Nebraska - Lincoln

USDA Wildlife Services - Staff Publications

U.S. Department of Agriculture: Animal and

Plant Health Inspection Service

2021

\title{
Estimating wildlife strike costs at US airports: A machine learning approach
}

Levi Altringer

Jordan Navin

Michael J. Begier

Stephanie A. Shwiff

Aaron M. Anderson

Follow this and additional works at: https://digitalcommons.unl.edu/icwdm_usdanwrc

Part of the Natural Resources and Conservation Commons, Natural Resources Management and Policy Commons, Other Environmental Sciences Commons, Other Veterinary Medicine Commons, Population Biology Commons, Terrestrial and Aquatic Ecology Commons, Veterinary Infectious Diseases Commons, Veterinary Microbiology and Immunobiology Commons, Veterinary Preventive Medicine, Epidemiology, and Public Health Commons, and the Zoology Commons

This Article is brought to you for free and open access by the U.S. Department of Agriculture: Animal and Plant Health Inspection Service at DigitalCommons@University of Nebraska - Lincoln. It has been accepted for inclusion in USDA Wildlife Services - Staff Publications by an authorized administrator of DigitalCommons@University of Nebraska - Lincoln. 


\title{
Estimating wildlife strike costs at US airports: A machine learning approach
}

\author{
Levi Altringer ${ }^{a, b, *, 1}$, Jordan Navin ${ }^{a}$, Michael J. Begier ${ }^{c}$, Stephanie A. Shwiff ${ }^{a}$, \\ Aaron Anderson ${ }^{a}$ \\ a US Department of Agriculture, Animal and Plant Health Inspection Service, Wildlife Services, National Wildlife Research Center, Fort \\ Collins, CO, 80521, USA \\ ${ }^{\mathrm{b}}$ Department of Economics, Colorado State University, Fort Collins, CO, 80523, USA \\ ${ }^{c}$ National Coordinator, Airport Wildlife Hazards Program, U.S. Department of Agriculture, Wildlife Services, WA, DC, 20250, USA
}

\section{A R T I C L E I N F O}

\section{Keywords:}

Wildlife strikes

Machine learning

Cost imputation

\begin{abstract}
A B S T R A C T
Current lower bound estimates of the economic burden of wildlife strikes make use of mean cost assignment to impute missing values in the National Wildlife Strike Database (NWSD). The accuracy of these estimates, however, are undermined by the skewed nature of reported cost data and fail to account for differences in observed strike characteristics-e.g., type of aircraft, size of aircraft, type of damage, size of animal struck, etc. This paper makes use of modern machine learning techniques to provide a more accurate measure of the strike-related costs that accrue to the US civil aviation industry. We estimate that wildlife strikes costed the US civil aviation industry a minimum average of $\$ 54.3$ million in total losses annually over the 1990-2018 period. If one assumes that wildlife strikes were underreported by as much as a factor of 3 over the same period, our estimates still fall below previous lower bound estimates.
\end{abstract}

\section{Introduction}

Costs associated with aircraft-wildlife collisions, or wildlife strikes, in the US are widely acknowledged by the aviation community (DeVault et al., 2011; Anderson et al., 2015; Dolbeer et al., 2019; Metz et al., 2020; Roca-González et al., 2020) and calculations of the economic burden of wildlife strikes to the US civil aviation industry rely on the reporting of strikes and cost information by aircraft operators and airport staff to the Federal Aviation Administration's (FAA) National Wildlife Strike Database (NWSD) (FAA, 2020). When reporting a wildlife strike to NWSD, the reporter is prompted to provide an estimate of (1) the repair costs as well as (2) the other costs associated with the strike. ${ }^{2}$ While repair costs are relatively intuitive, costs that can be considered as other are less straight forward. The FAA's wildlife strike reporting form indicates that "revenue loss, fuel, aircraft inspection, crew lodging or rescheduling, etc." are examples of costs that fall under the category of other. Other costs, then, are costs that are only indirectly associated the wildlife strike-for this reason some have referred to reported costs as either direct or indirect. Historically, reported total costs are calculated to be the sum of repair and other costs reported to the NWSD (Dolbeer et al., 2019).

\footnotetext{
This research was supported by the U.S. Department of Agriculture, Animal and Plant Health Inspection Service, Wildlife Services, National Wildlife Research Center.

* Corresponding author at: US Department of Agriculture, Animal and Plant Health Inspection Service, Wildlife Services, National Wildlife Research Center, Fort Collins, CO, 80521, USA.

E-mail address: levi.altringer@colostate.edu (L. Altringer).

1 Data and code for replication can be accessed at https://github.com/levialtringer/machine_learning_wildlife_strike_cost_estimates.

2 The FAA's Form 5200-7 can be found at https://www.faa.gov/documentLibrary/media/form/faa5200-7.pdf and the electronic version can be found at https://wildlife.faa.gov/add.
}

https://doi.org/10.1016/j.trd.2021.102907

Available online 27 May 2021

1361-9209/C 2021 Elsevier Ltd. All rights reserved. 


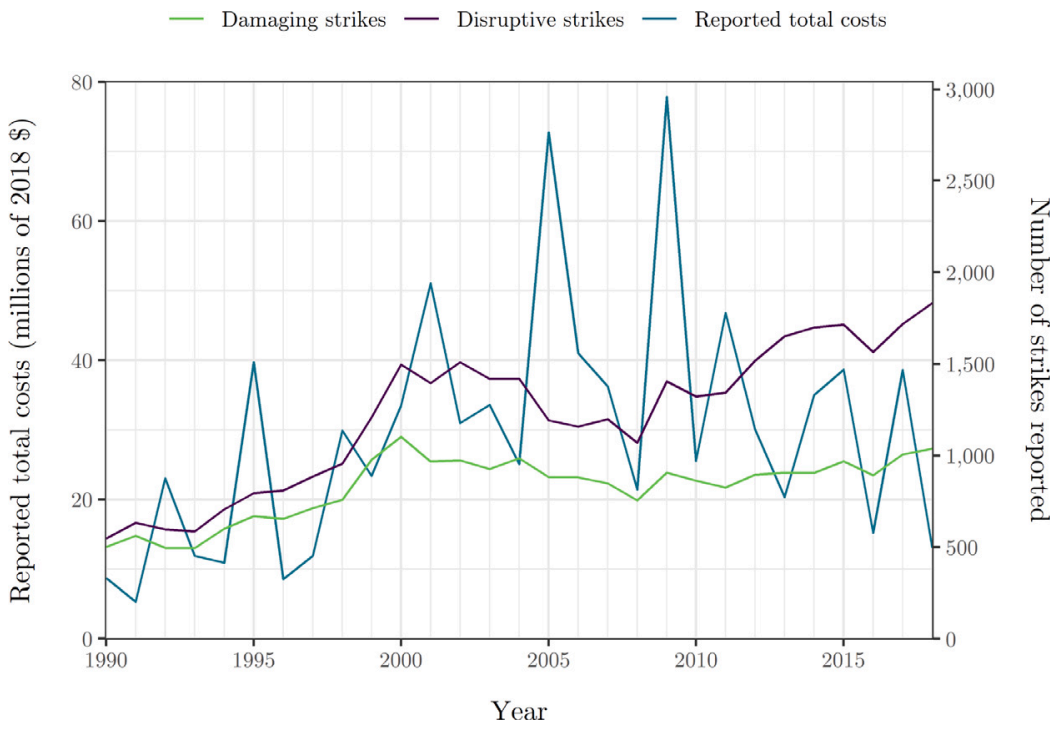

Fig. 1. Strikes and total costs reported in the NWSD, 1990-2018.

Fig. 1 plots the trend in reported total costs, as well as the number of reported disruptive and damaging strikes, over the 19902018 period. Here, a disruptive strike is one that reported one or more of the following: repair cost, other cost, damage, a negative effect on the flight, or downtime. This is similar to (Dolbeer et al., 2019)'s definition of "adverse incidents". A damaging strike is simply a strike that reported structural damage to the aircraft. As one might expect, total costs reported to the NWSD follow a trend similar to that of reported disruptive and damaging strikes, though reported total costs tend to be much more variable from year to year due to the intermittent presence of rare, extremely high-cost incidents. Over the 1990-2018 period, an average of $\$ 29.7$ million (2018 \$) in real annual monetary losses were reported to the NWSD. From 2000 to 2018, a period when the reporting of disruptive and damaging strikes was relatively stable, average annual reported monetary losses were $\$ 36.2$ million.

There are multiple reasons why the reported total costs derived from the NWSD significantly underestimate the economic burden of aircraft-wildlife strikes to the US civilian aviation industry. First, not all wildlife strikes reported to the NWSD provide cost information. Of the 24,967 strike incidents that indicate damage to the aircraft, only 5,129 (20.5\%) have non-missing repair cost information and only 5,860 (2.5\%) of the total 237,305 wildlife strikes reported to the NWSD provide information concerning the other costs associated with the strike (FAA, 2020). Second, aircraft-wildlife collisions have a history of being underreportedespecially in the beginning of the 1990-2018 period. Previous research indicates that less than 20 percent of all strikes were reported by US airports and airlines over the 1990-2003 period, and that only 39 and 47 percent of strikes were reported at part 139 airports in 2004-2008 and 2009-2013, respectively (Cleary and Dolbeer, 2005; Dolbeer, 2015). Third, the costs reported to the NWSD pertain only to the US civil aviation industry and, therefore, provide no measure of external costs that fall on others affected by wildlife strikes-e.g., costs associated with delays imposed on passengers and the transport of goods and services. Lastly, an additional issue is the reliability of the cost data that is reported to the NWSD, which may be (1) an imperfect approximation by pilots and airport staff and (2) influenced by strategic behavior among airline companies (Navin et al., 2020).

Limited by the issues of underreporting and intangible external costs, previous studies have attempted to generate a more accurate lower bound estimate of total costs by imputing cost values to reported strikes with missing cost information in the NWSD. The most recent lower bound cost estimates were produced by Dolbeer et al. (2019), who use mean cost assignment to address the problem of missing information. Using mean cost assignment to impute missing costs in the NWSD, however, comes with a number of disadvantages given the skewed nature of reported cost data. Moreover, these estimates do not account for differences in observed strike characteristics - e.g. type of aircraft, size of aircraft, type of damage, size of animal struck - that allow for a more accurate estimate of strike-related costs.

In this paper we exploit the presence of cost-relevant information provided for each strike report in the NWSD and employ modern machine learning techniques to provide a more accurate lower bound estimate of monetary losses that accrue to the US civil aviation industry as a result of wildlife strikes. Ultimately, we test the accuracy of two supervised machine learning algorithms random forest and artificial neural network - against linear regression and find that modern machine learning techniques outperform traditional econometric methods when imputing repair and other costs in the NWSD. Then, employing the best performing model we impute missing costs in the NWSD and estimate that wildlife strikes generated a minimum of $\$ 54.3$ million in real annual monetary losses to the US civil aviation industry over the 1990-2018 period. The cost estimates generated by our models are almost entirely unaffected by the rare, extremely high-cost incidents that undermine the accuracy of mean cost assignment estimates. Even after taking into account the issue of underreporting, our estimates suggest that previous estimates may actually be a reasonable upper, not lower, bound approximation of strike-related costs borne by the US civil aviation industry. We conclude with a discussion of the implications for accurately measuring the benefits of wildlife management at airports and efficiently allocating wildlife management resources across airports. 


\section{Background and motivation}

As stated above, the most recent lower bound estimates of the economic burden of wildlife strike events were produced by Dolbeer et al. (2019), who use mean cost assignment to impute missing cost information in the NWSD. Specifically, for each year of the 19902018 period, mean reported repair and other costs are calculated using strike reports with non-missing cost information. These mean cost calculations are then multiplied by the total number of disruptive strikes that occurred in their respective years to obtain yearly lower bound estimates of total costs. Using this method, Dolbeer and coauthors estimate that minimum yearly average monetary losses to the US civil aviation community were \$187 million over the 1990-2018 period and write that "actual costs are likely 2 or more times higher" due to the issue of underreporting.

Using mean cost assignment to impute missing costs in the NWSD, however, comes with a number of disadvantages. First, the assumption has to be made that all reported wildlife strikes, at least the ones that can be considered disruptive, engendered similar amounts of damage and downtime, among other adverse effects. Thus, wildlife strikes that vary significantly with respect to the aircraft type, damage type, etc., are assigned equal costs via mean cost imputation. Second, imputation through mean cost assignment is problematic when data are skewed-the summary statistics presented in Table 1 of Section 4.1 indicate that reported costs in the NWSD are skewed positively. The following example makes this point clear within the context of this study.

Consider the year 2009, where 206 strike incidents reported total real repair costs of $\$ 77,852,306$ and 524 strike incidents reported total real other costs of $\$ 2,897,579$ (2018 $\$$ ). Then, the mean total cost per strike is calculated to be $[\$ 77,852,306 / 206+$ $\$ 2,897,579 / 524]=\$ 383,453$. Applying this mean to the 1,405 disruptive strike incidents in 2009 , we obtain a lower bound total cost estimate of $\$ 538,751,465$. However, if we remove the "Miracle on the Hudson" strike incident - when US Airways Flight 1549 ditched into the Hudson River - from the 2009 data, the lower bound total cost estimate falls by $42 \%$ to $\$ 310,231,388$. In other words, the removal of one strike incident, which by itself generated $\$ 42,117,878$ in real monetary losses, reduces the lower bound total cost estimate by $\$ 228,520,077$. The US Airways Flight 1549 incident is an extreme case, but the accuracy of mean cost assignment is similarly sensitive to the presence of less extreme low-probability, high-cost incidents. Therefore, a cost imputation method that makes use of cost-relevant strike characteristics and is robust to the presence of rare, high-cost strike incidents can provide an improved lower bound estimate of the economic burden of wildlife strikes to the US civil aviation industry.

The drawbacks associated with previous methods of cost imputation are not the only motivation for improving upon the estimates generated by previous research. First, in so far as wildlife management activities are effective in reducing the number and intensity of strike incidents that occur at a given airport, an accurate measure of the benefit of these management activities requires that one has an accurate estimate of strike-related costs. Robust estimates of the costs associated with wildlife strikes, then, provides a first step in precisely measuring the benefits of wildlife management at airports. Moreover, accurate cost estimates can provide a measure of relative risk at airports beyond relative measures of strikes, disruptive strikes, and damaging strikes. For instance, though two airports may have similar levels of risk as measured by disruptive and damaging strikes per movement, improved cost estimates may determine that the cost per damaging strike at one airport is relatively large compared to others and lend itself to a more efficient allocation of publicly-funded wildlife management resources.

\section{Methods}

While statistical models such as Anderson et al. (2015)'s may be the optimal choice for gaining inference related to the specific factors that influence the probability and cost of a wildlife strike, supervised machine learning algorithms have emerged as a useful method for the problem of prediction, which lies at the center of this article (Mannering et al., 2020). In the context of transportation related accidents, machine learning techniques have been shown to be a useful tool when predicting the frequency and intensity of road traffic incidents, outperforming more traditional statistical methods (Tang et al., 2020; Mannering and Bhat, 2014; Xie et al., 2007; Chang, 2005; Abdelwahab and Abdel-Aty, 2001). Relatedly, Riviere et al. (2006) show that a Bayesian Neural Network provides a precise estimate of a vehicles energy equivalent speed - a continuous target variable as opposed to a count variable - at impact, making use of observed features that contain information about both the vehicle and accident.

Further, our study is not the first to make use of supervised machine learning algorithms to predict cost-related data. In the presence of many input variables, Mullainathan and Spiess (2017) find several machine learning algorithms, including random forest, outperform ordinary least squares when predicting home values in the 2011 American Housing Survey. Verlinden et al. (2008) estimate the costs of sheet-metal production projects by feeding various variables, including material type, sheet thickness, and the number of holes, through an artificial neural network in addition to using traditional econometric techniques. They show that at the expense of inference, using a neural network significantly improved prediction performance.

Generally, supervised machine learning algorithms seek to predict a target value $(y)$ from its observed features ( $x$ ) via searching for a function $\hat{f}$ that minimizes a specified loss function $L(\hat{f}(x), y)$ on a new data point from the same distribution (Mullainathan and Spiess, 2017). In the context of the wildlife strike cost problem, $x$ is various characteristics of the strike - including aircraft type, the size of the animal struck, the phase of flight in which the strike occurred, etc. - while $y$ is repair costs or other costs. For classification machine learning problems, the loss function is generally derived from the share of incorrectly predicted outcomes. In the context of regression, however, mean square error (MSE) and mean absolute error (MAE) are often utilized as loss functions depending on one's preference to place equal weight on all errors (MAE) or more weight on larger errors (MSE). We specify MSE as the loss function during model training and evaluate the performance each model on test set data using MSE, MAE, and R-squared.

The question then becomes, which learning algorithm and model can best predict the cost of a wildlife strike from its observed features? We compare the performance of two supervised machine learning algorithms, a random forest and artificial neural network, 
Table 1

Repair and other cost summary statistics.

\begin{tabular}{|c|c|c|c|c|c|c|}
\hline & Mean & Std Dev & Min & Median & Max & $N$ \\
\hline \multicolumn{7}{|l|}{ Repair costs } \\
\hline \multicolumn{7}{|c|}{$(19,838$ missing values)* } \\
\hline \multicolumn{7}{|l|}{ Actual: } \\
\hline All & $\$ 152,646$ & $\$ 926,856$ & $\$ 1.02$ & $\$ 13,670$ & $\$ 42,117,878$ & 5,129 \\
\hline Training & $\$ 156,900$ & $\$ 968,301$ & $\$ 1.02$ & $\$ 13,557$ & $\$ 42,117,878$ & 4,103 \\
\hline Test & $\$ 135,632$ & $\$ 738,383$ & $\$ 1.41$ & $\$ 13,936$ & $\$ 16,997,757$ & 1,026 \\
\hline \multicolumn{7}{|l|}{ Log: } \\
\hline All & 9.40 & 2.34 & 0.02 & 9.52 & 17.56 & 5,129 \\
\hline Training & 9.41 & 2.35 & 0.02 & 9.51 & 17.56 & 4,103 \\
\hline Test & 9.38 & 2.31 & 0.35 & 9.54 & 16.65 & 1,026 \\
\hline \multicolumn{7}{|l|}{ Other costs } \\
\hline \multicolumn{7}{|c|}{ (231,445 missing values) } \\
\hline \multicolumn{7}{|l|}{ Actual: } \\
\hline All & $\$ 16,225$ & $\$ 149,036$ & $\$ 0.01 * *$ & $\$ 234$ & $\$ 6,419,450$ & 5,860 \\
\hline Training & $\$ 15,770$ & $\$ 148,957$ & $\$ 0.01$ & $\$ 235$ & $\$ 6,419,450$ & 4,688 \\
\hline Test & $\$ 18,049$ & $\$ 149,403$ & $\$ 0.01$ & $\$ 230$ & $\$ 4,370,334$ & 1,172 \\
\hline \multicolumn{7}{|l|}{ Log: } \\
\hline All & 3.37 & 5.84 & -4.61 & 5.46 & 15.67 & 5,860 \\
\hline Train & 3.36 & 5.86 & -4.61 & 5.46 & 15.67 & 4,688 \\
\hline Test & 3.43 & 5.78 & -4.61 & 5.44 & 15.29 & 1,172 \\
\hline
\end{tabular}

NOTE: *The assumption is made that all non-damaging strikes reported to the NWSD $(N=212,338,89.5 \%)$ have a repair cost of $\$ 0$. Thus, the repair cost imputation exercise only pertains to damaging strikes $(N=24,967,10.5 \%)$. This distinction is not made for the other cost imputation exercise, which is why the number of missing other costs values to predict is substantially larger. **Further, our log transformation of the target variable requires that we replace other cost values of $\$ 0$ with $\$ 0.01$.

against ordinary least squares regression, seeking to provide a more robust lower bound estimate of the economic burden of wildlife strikes to the US civil aviation industry. All analyses were done with R software (version 3.6.1). In the sections that follow we discuss the data partitioning methods required to robustly evaluate the absolute and relative predictive performance of each algorithm and provide a foundational discussion of the random forest and artificial neural network algorithms. The particular specifications of the models used in our final model testing and cost imputation exercises are presented in the beginning of Section 5.

\subsection{Model training, testing, selection, and prediction}

Evaluating the absolute and relative performance of a machine learning algorithm requires a partitioning of the data into a training and test set, or what is known as the holdout method (Step 1, Fig. 2) (Raschka, 2018). As the name suggests, the training set is used to train the learning algorithm(s), a process which involves tuning algorithm-specific hyperparameters which will be discussed in the sections that follow. The test set, which is unseen by the learning algorithm(s) during the training stage, is used in a final stage to evaluate the absolute and relative predictive performance of each algorithm on out-of-training-sample data. The purpose of the holdout method is to combat the empirical problem of overfitting, which occurs when a learning algorithm's performance on in-sample data overstates its ability to predict unseen, out-of-sample data-also known as optimistic bias. On the other hand, the holdout method introduces pessimistic bias - when algorithm performance on in-sample data understates its ability to predict out-of-sample data - due to the fact that the performance of each learning algorithm is limited by the amount of data available to learn from. Taking this tradeoff into account, we perform a 80/20 train-test random split of the data in an attempt to limit both optimistic and pessimistic bias from influencing our evaluation of absolute and relative algorithm performance, model selection, and, ultimately, our lower bound estimates of the economic burden of wildlife strikes.

During the training stage of analysis, algorithm-specific hyperparameters are tuned in an effort to find the best-performing model for each learning algorithm. Each time the value of a hyperparameter is changed, a new model is generated. A model is a subclass of a learning algorithm, defined by a unique combination of hyperparameters. Here, the same empirical problem of overfitting the training data applies, where one combination of hyperparameter values may perform relatively well on the training set, but fail to perform on out-of-training-sample data. We employ a technique called cross-validation, specifically 10-fold cross-validation, for hyperparameter tuning in the training stage of analysis to, again, limit the influence of optimistic and pessimistic bias in model selection (Step 2, Fig. 2) (Raschka, 2018). During 10-fold cross-validation, a model's performance is measured as the average performance across each fold of the training set (Step 3, Fig. 2) and hyperparameter tuning is repeated for each algorithm until the best performing combination of hyperparameters, or model, is found and selected (Step 4, Fig. 2). Specifically, we define a search space as a grid of hyperparameter values and evaluate every position in the grid, selecting the best performing combination of hyperparameter values as our final model specification for each algorithm.

Once the best performing model has been selected via grid search for each algorithm, the test set is then used to measure absolute and relative model performance. In this step, we perform 100 random samples (without replacement) on the test set to generate a distribution of accuracy metrics that characterize the performance of each model on unseen, out-of-training-sample data. From the distribution of these metrics the mean and standard deviation of model performance can be calculated and compared to inform the 


\section{NWSD}

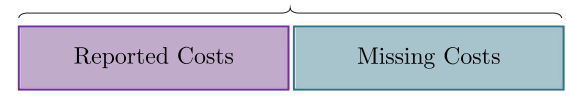

Step 1: Train/Test Split

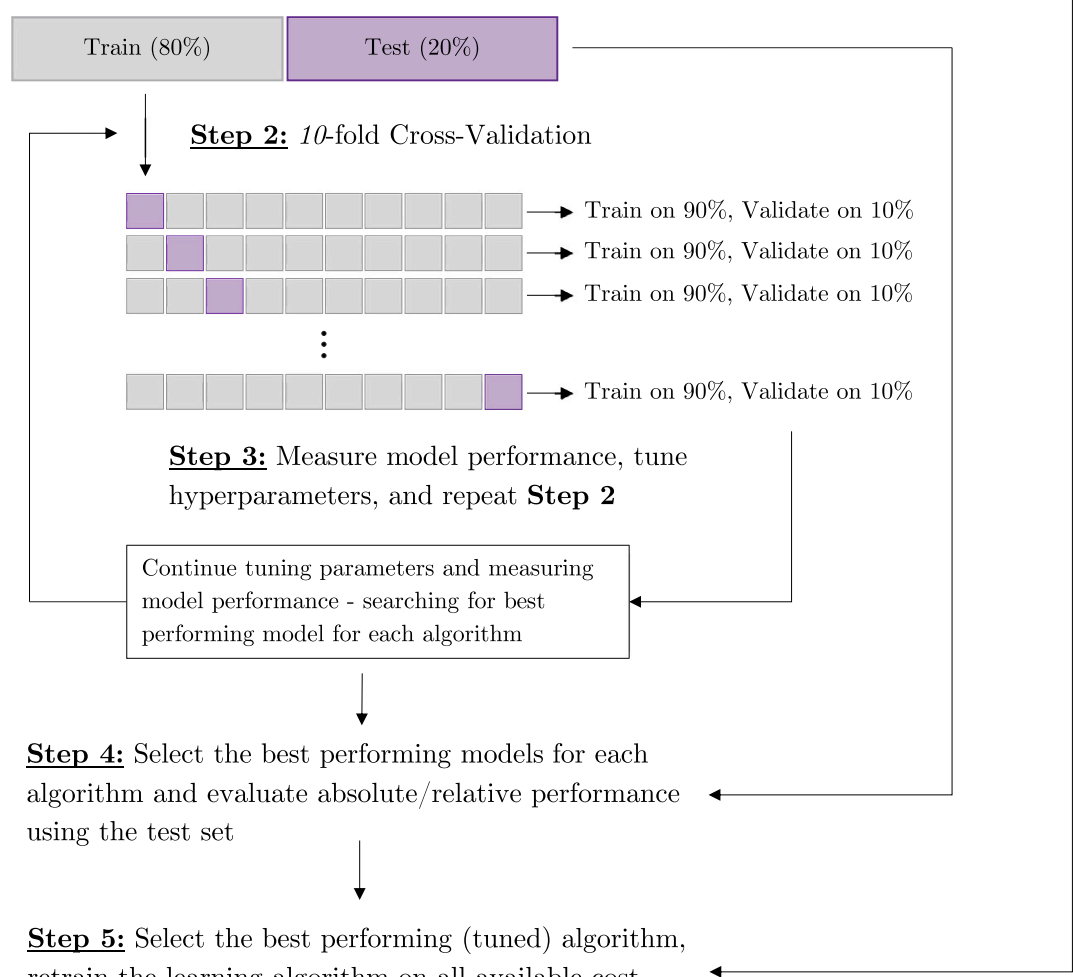
retrain the learning algorithm on all available cost data, and predict missing costs in the NWSD

Fig. 2. Workflow of model training, model testing, and imputing missing costs. NOTE: Step 2 is repeated iteratively for each learning algorithm until the best performing model is found. In Step 4, each (tuned) algorithm is tested on 100 random samples of the test set-generating the distribution of performance metrics shown in Figs. 5 and 6.

selection of the final model to be used in the prediction of missing costs in the NWSD (Step 5, Fig. 2). In the final stage of analysis we combine the reported cost data with imputed cost data to generate yearly lower bound estimates of costs that accrue to the US aviation industry as a result of aircraft-wildlife collisions (Step 6, Fig. 2).

\subsection{Random forest}

Random forest is a non-parametric supervised machine learning algorithm rooted in regression trees. Regression trees are comprised of layers of nodes - each node characterized by an explanatory feature in the data set - and edges-where the number of edges emanating from each node is determined by each feature's range of values. Consider a simple example with two explanatory features - whether the animal struck was ingested into the engine or not (INGESTED) and whether the animal struck was large or not (ANIMAL "LARGE") - both of which are binary indicator variables and, therefore, have only two edges emanating from each node (Fig. 3). Observations pass through each node and down the edges of the tree with their path being determined by the value of their observed features-e.g., if the animal struck was ingested into the engine (right) or not (left) and if the animal struck was large (right) or not (left) (Fig. 3). Thus, a regression tree successively partitions the data into increasingly homogeneous subsets according to each 


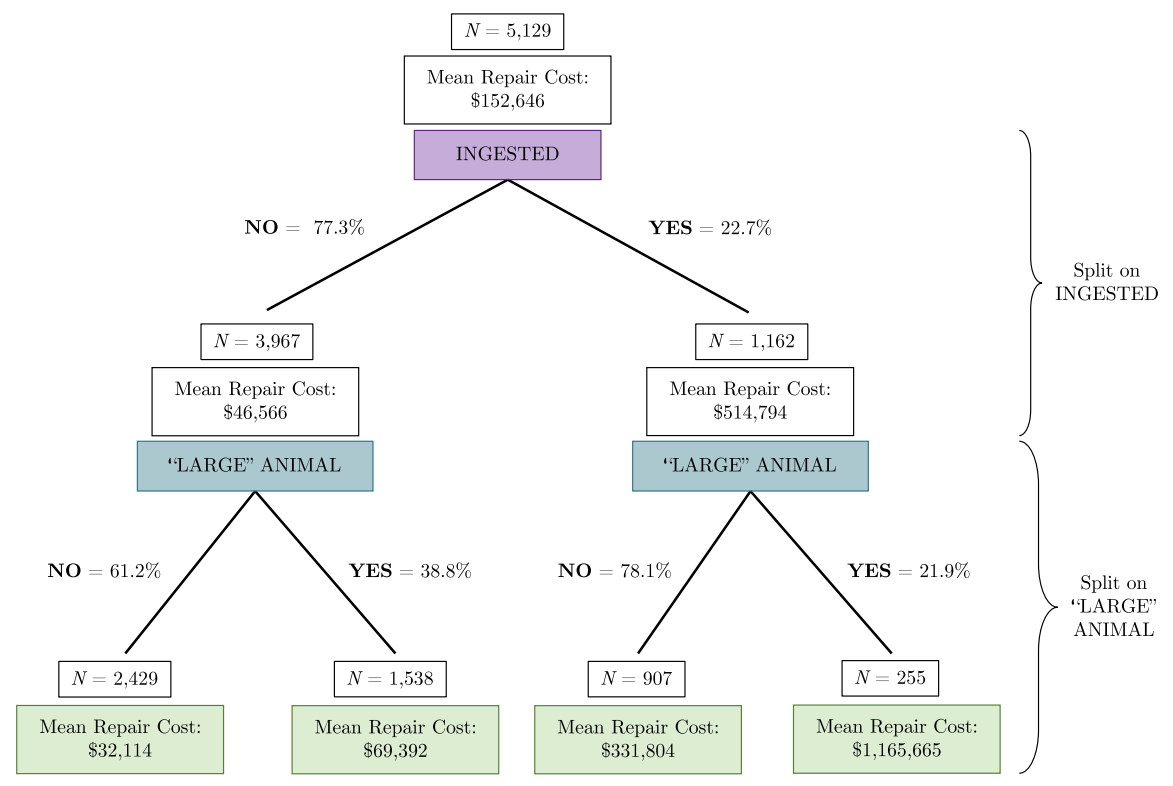

Fig. 3. Repair cost regression tree with two explanatory features.

observation's observed features. Ultimately, a terminal node is reached and a prediction is given by averaging the target variablee.g., repair cost-for the subset of observations at each terminal node. As an aside, this simple example provides preliminary evidence that the explanatory features given in the NWSD can account for substantial variation in reported repair costs-see Fig. 3.

There are a number of factors that determine the ultimate structure of a regression tree (Moisen, 2008), we discuss two here. First, a splitting rule is enforced that determines (1) which explanatory features should be split on first, second, third, and so on, and (2) whether or not a regression tree will split on any given explanatory feature at all. The purpose of the splitting rule is to minimize node impurity or, put another way, to ensure that the most important features are placed closer to the root of the tree and that the predictive power of the tree is increasing monotonically as one moves down the tree. The splitting rule used in our analysis is mean square error (MSE), meaning that (1) the explanatory features placed near the root of the tree provide the largest reduction in MSE relative to other features and (2) a split on a certain explanatory feature will only occur if the resulting MSE is lower than that given by the previous node. The regression tree shown in the previously discussed example (Fig. 3) implies that initially splitting on INGESTED provides a lower MSE than splitting on "LARGE" ANIMAL. It also implies that subsequently splitting on "LARGE" ANIMAL, after previously splitting on INGESTED, reduces MSE and improves the regression tree's predictive performance.

Second, one must determine the appropriate depth of a regression tree. A tree can be grown quite large, so much so that it fits the training data almost perfectly with a very small amount of observations in each terminal node but generates poor predictions on unseen, out-of-training-sample data. This is certainly not the case in our example since the depth of the tree is limited by the small number of explanatory features included. However, if the number of explanatory features is large, then limiting the depth of a tree can combat the empirical problem of overfitting. This is achieved by setting a minimum number of observations that are required at each terminal node, $n$.

Finally, the random forest machine learning algorithm is an ensemble method that, building on the basic structure of a regression tree, seeks to combat the empirical problem of overfitting (Moisen, 2008). First, a bootstrap sample of the training data is selected. Then, at the root node, a random sample of $f$ explanatory features is drawn and the best split is made from the sample of the subset of features. At each successive node, another random sample of explanatory features is selected and the best split is made. The tree continues to grow until maximum depth is achieved, with depth being determined by the minimum number of observations required at each terminal node, $n$. This process is repeated $t$ times, where $t$ is the specified number of trees that algorithm is told to grow. Once all $t$ trees have been grown, the prediction for each observation is calculated as the average prediction across all relevant terminal nodes in the forest. There are three hyperparameters mentioned in the discussion above that we tune via grid search during the training stage of analysis. These are the minimum number of observations required at each terminal node of each tree $(n)$, the number of explanatory features to randomly draw and test for each split $(f)$, and the number of trees to grow $(t)$. The random forest learning algorithm is implemented using the randomForest package (version 4.14) in $\mathrm{R}$ (Liaw et al., 2002).

\subsection{Artificial neural network}

An artificial neural network - or simply neural network - is a complex, parametric supervised machine learning algorithm composed of several layers organized hierarchically. Similar to the random forest algorithm, a neural network is best explained through a preliminary example (Fig. 4). A neural network reads left to right, beginning with the input layer. Similar to the random 


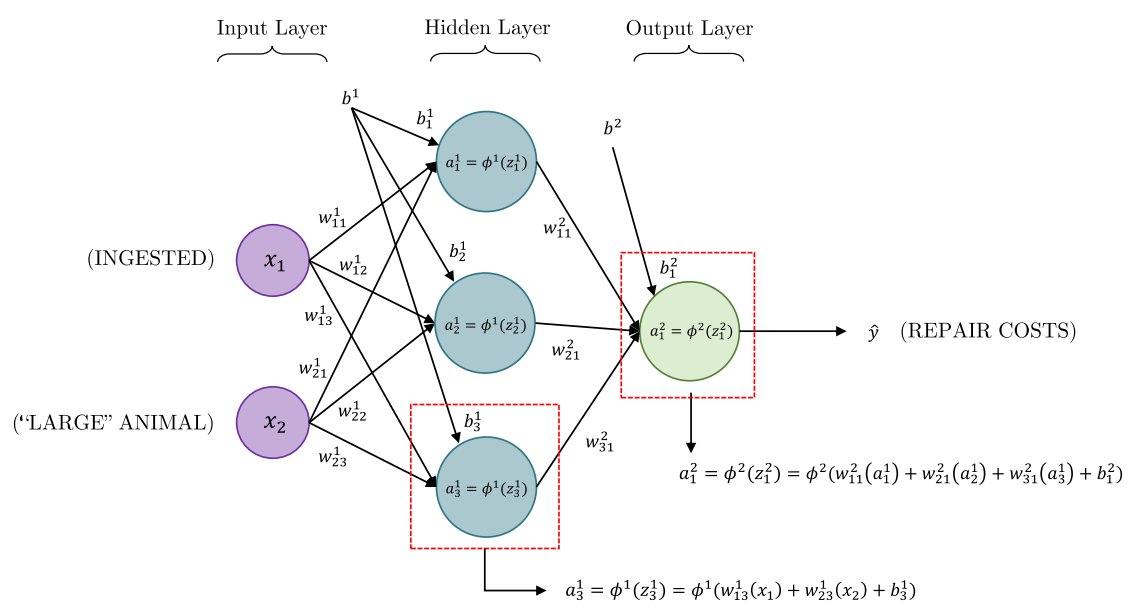

Fig. 4. Repair cost neural network with two input features and one hidden layer.

forest example, the two explanatory features selected as inputs into the network are whether the animal struck was ingested into the engine or not $\left(x_{1}\right)$ and whether the animal struck was large or not $\left(x_{2}\right)$. The input matrix $(x)$ in this neural network has dimensions $2 \times n$ where input features are indexed vertically (rows) and the number of training observations $n$ is indexed horizontally (columns). While traveling from the input layer to the neurons in the hidden layer, the data is transformed into a linear combination of the inputs $\left(z_{i}^{1}\right)$ via a weighting matrix and a neuron specific constant called a bias term $\left(w^{1} x+b^{1}\right)$. Here, $w^{1}$ is a matrix with dimensions $3 \times 2$ that correspond to the three hidden layers and two input features. The bias matrix, $b^{1}$, has dimensions $3 \times n$ corresponding to the three hidden layers and number of training observations where, for each row, the same bias term is distributed across the columns of the bias matrix. The result of this transformation is a $3 \times n$ matrix, $z^{1}$, in the first hidden layer that contains the neuron-specific linear combinations of the input data.

Before moving to the subsequent layer, the $z^{1}$ matrix is transformed nonlinearly through a chosen activation function $\phi^{1}$ to deliver a matrix of values $a^{1}$ - with dimensions $(3 \times n)$ - to be passed on to each unit in the following layer. In our analysis, all activation functions are a rectified linear unit (ReLU), except in the output layer, which is linear due to the nature of the prediction. ReLU is one of the most commonly used activation functions in deep learning, transforming all elements of $z^{1}$ to positive values such that $a_{i j}^{1}=f\left(z_{i j}^{1}\right)=\max \left(0, z_{i j}^{1}\right)$ (Ramachandran et al., 2017). The transformed and activated data then travels from the hidden layer to the output layer where it is again transformed into a linear combination $\left(z^{2}\right)$ of neuron outputs through a second weighting matrix $\left(w^{2}\right)$ - which is a $1 \times 3$ vector in this example - and augmented with a second bias term $\left(w^{2} a^{1}+b^{2}\right)$. The $z^{2}$ matrix, which is simply a vector in this example, is then transformed through a linear activation function $\left(\phi^{2}\right)$ in the output layer due to the continuous nature of the target variable. The result of the final transformation in the output layer is $(1 \times n)$ vector $\mathrm{a}^{2}$ that constitutes that network's repair cost predictions $(\hat{y})$ for the $n$ training observations.

The objective of the neural net is to generate estimates of $w$ and $b$ so as to minimize prediction error, or loss, which we specify to be mean squared error (MSE). The relatively simple neural network discussed above (Fig. 4) is an example of what is called a "feed-forward" network, meaning the flow of information only moves from the inputs (left) to the output (right). However, backpropagation allows the algorithm to learn from the prediction errors $(y-\hat{y})$ realized in the output layer. Backpropagation sends the errors backward through the network to update the estimates of $w$ and $b$ using the process of gradient descent, where partial derivatives of the loss function with respect to $w$ and $b$ determine the direction of update. One pass forward via forward propagation and backward using gradient descent through the network is a single iteration, or epoch, and this process is repeated iteratively to find the entire set of parameters $w$ and $b$ that minimize the loss function.

Similar to a random forest, several hyperparameters are tuned via grid search in the model training stage to improve the performance of a neural network. These hyperparameters include the number of hidden layers in the network, the number of neurons in each hidden layer, and the number of iterations to go forward and backward through the network, or epochs. Further, dropout rules can be employed after each hidden layer where a randomly selected set of neurons are ignored, or temporarily removed from the layer, before the data is passed to the next within each epoch. Similar to the random processes employed in the random forest algorithm, dropout rules within a neural network attempt to combat the empirical problem of overfitting. The artificial neural network learning algorithm is implemented using the keras package (version 2.2.5.0) in R (Arnold, 2017). 


\section{Data}

\subsection{Repair and other costs}

The NWSD currently contains records of 237,305 voluntarily reported aircraft-wildlife strikes that occurred at non-military airports (FAA, 2020). ${ }^{3}$ Upon inspection of the data, there are 64 strike reports from the 1982-1989 period and 14,260 reports from 2019. We include these reports in model training and testing as they allow each learning algorithm to see more data, improve their predictive capabilities, and allow for a more robust evaluation of model performance. However, we reduce the sample to the 1990-2018 period in the final presentation of yearly estimates since strikes from 1982-1989 are severely underreported and not all reports from 2019 have been entered into the NWSD.

Costs are reported to the NWSD in two categories-repair and other costs. Beginning with repair costs, of the 237,305 wildlife strikes reported in the NWSD, 212,338 (89.5\%) indicate that the strike resulted in no damage to the aircraft while 24,967 (10.5\%) report damage. After consulting with the Wildlife Project Lead at the FAA Technical Center and the NWSD data scrubbing team, we make the assumption that all non-damaging strikes have a repair cost of zero. We see this as an intuitive and also reasonable assumption given that, among the 212,338 non-damaging strikes, 212,335 do not report a repair cost while 3 report a cost of zero. Of the 24,967 strike incidents that report damage, 5,129 (20.5\%) have non-missing repair cost and 19,838 (79.5\%) have missing information. Real reported repair costs vary widely (Mean $=\$ 152,646, \mathrm{SD}=\$ 926,856)$ and are skewed positively $($ Min $=\$ 1$, Median $=\$ 13,670$, Max $=\$ 42,117,878$ ) (Table 1). As discussed in Section 3.1, the 5,129 damaging strike reports with non-missing repair cost information are divided into a training set $(80 \%)$ and test set $(20 \%)$. The summary statistics for these sets indicate that they are both representative of the overall distribution of non-missing repair costs (Table 1 ).

Moving to other costs, only 5,860 (2.5\%) of the 237,305 wildlife strikes reported in the NWSD provide information about other costs associated with the strike. Given that damage is not directly related to non-repair costs, we do not assume that non-damaging strikes have an other cost of $\$ 0$. Reported other costs are significantly smaller than repair costs (Mean $=\$ 16,225, \mathrm{SD}=\$ 149,036)$, but are similarly positively skewed (Min $=\$ 0$, Median $=\$ 234$, Max $=\$ 6,419,450$ ) (Table 1 ). The 5,860 strike reports with nonmissing other cost information are partitioned into a training set $(80 \%)$ and test set $(20 \%)$ that are both representative of the overall distribution of non-missing other costs. Log repair and other costs will serve as the target variables in our model training, model testing, and missing cost imputation-similar to Mullainathan and Spiess (2017) whose target variable is log home values. Transforming the data in this way removes, or reduces, the skewness of the cost data - see Table 1 - which improves the predictive capabilities of our models without influencing our evaluation of relative model performance (Jiang et al., 2008). The data is transformed back into dollar values in the final presentation of our estimates.

\subsection{Explanatory features}

The NWSD contains information on over 90 different variables associated with each individual strike. The explanatory features $(x)$ used in our analysis were selected in an effort to encompass as many factors that may influence the cost of a wildlife strike as possible (Table 2)—similar to Anderson et al. (2015). The features selected include: aircraft class, the engine type of the aircraft, aircraft mass, whether or not the pilot was warned of birds or wildlife prior to the strike, the phase of flight when the strike occurred, the number of animals seen by the aircraft's pilot, the number of animals struck, the size of the animal(s) struck, the effect of the strike on the flight, the type (or intensity) of damage reported, whether or not the animal was ingested by the aircraft's engine, cloud cover, and time of day. Individual dummy variables for the specific aircraft component struck and the specific aircraft component damaged are also included. The models of other costs also include an indicator variable for whether or not the strike resulted in damage to the aircraft, in addition to the type of damage indicator mentioned above. This feature is not included in the models of repair costs since all strike reports with non-zero and missing repair costs are damaging.

\section{Results and discussion}

\subsection{Model evaluation and selection}

From the training stage of modeling, four tuned algorithms, or models, emerge. Two random forest models—one for repair costs and another for other costs-and two neural network models-again, one for repair costs and the second for other costs. Again, during the training stage we perform a grid search of hyperparameter values to determine which combination provides the most accurate prediction of the training cost data.

The random forest hyperparameters selected are the same for both the repair and other cost models, with the number of trees in the forest set to 300 , the number of explanatory features randomly selected as potential split candidates set to 20 , and the minimum number of observations required at each terminal node set to 20. The repair cost neural network is composed an input layer with 95 inputs, two hidden layers - with 75 neurons in the first hidden layer and 55 neurons in the second - and an output layer. Rectified linear activation (ReLU) is the activation function specified for each hidden layer and linear activation is used in the output layer due to the continuous nature of the target variable. The number of epochs is set to 20, meaning that the input data (passed forward)

3 NWSD was last accessed on April 15th, 2020 (FAA, 2020). 
Table 2

Variables used as explanatory features in the imputation of missing costs.

\begin{tabular}{|c|c|c|}
\hline Variable & Variable type & Description \\
\hline Aircraft class & Categorical & Airplane; Helicopter; Glider; Ultralight; Missing \\
\hline Engine type & Categorical & $\begin{array}{l}\text { Reciprocating engine (piston); Turbofan; Turbojet; Turboprop; } \\
\text { Turboshaft (helicopter); Missing }\end{array}$ \\
\hline Aircraft mass & Categorical & $\begin{array}{l}2250 \mathrm{~kg} \text { or less; } 2251-5700 \mathrm{~kg} ; 5701-27,000 \mathrm{~kg} ; 27,001-272,000 \\
\mathrm{~kg} \text {; above } 272,000 \mathrm{~kg} \text {; Missing }\end{array}$ \\
\hline Pilot warned & Categorical & No; Yes; Missing \\
\hline Phase of flight & Categorical & $\begin{array}{l}\text { Approach; Arrival; Climb; Departure; Descent; En route; Landing } \\
\text { roll; Local; Parked; Taxi; Take-off run; Missing }\end{array}$ \\
\hline Number seen & Categorical & $1 ; 2-10 ; 11$ or more; Missing \\
\hline Number struck & Categorical & $1 ; 2-10 ; 11$ or more; Missing \\
\hline Animal size & Categorical & Small; Medium; Large; Missing \\
\hline Component struck & Dummies & $\begin{array}{l}\text { A dummy variable for each of the following components of the } \\
\text { aircraft: radome, windshield, nose, engine } 1 \text {, engine } 2 \text {, engine } 3 \text {, } \\
\text { engine } 4 \text {, propeller, wing or rotor, fuselage, landing gear, tail, } \\
\text { lights, other }\end{array}$ \\
\hline Effect on flight & Categorical & $\begin{array}{l}\text { Aborted takeoff; Engine Shutdown; Precautionary landing; Other; } \\
\text { None; Missing }\end{array}$ \\
\hline Damage* & Categorical & No; Yes \\
\hline Damage type & Categorical & None; Minor; Substantial; Destroyed; Uncertain; Missing \\
\hline Component damaged & Dummies & $\begin{array}{l}\text { A dummy variable for each of the following components of the } \\
\text { aircraft: radome, windshield, nose, engine } 1 \text {, engine } 2 \text {, engine } 3 \text {, } \\
\text { engine } 4 \text {, propeller, wing or rotor, fuselage, landing gear, tail, } \\
\text { lights, other }\end{array}$ \\
\hline Ingested & Categorical & No; Yes; Missing \\
\hline Cloud cover & Categorical & No clouds; Some clouds; Overcast; Missing \\
\hline Time of day & Categorical & Dawn; Day; Dusk; Night; Missing \\
\hline
\end{tabular}

NOTE: *The damage variable is not used as an explanatory feature in models of repair cost for the reasons outlined in Section 4.1.

and predictions errors (passed backward) will travel through the network 20 times with the estimated weights ( $w$ ) and bias terms (b) being updated in each epoch. The other cost neural network model is nearly identical to the model just described for repair costs, with the two exceptions being that (1) the first hidden layer is composed of 96 neurons, not 75, and (2) a 5 percent dropout rule is employed in each hidden layer to combat overfitting. The performance of these models are compared to that of ordinary least squares regression, where the same explanatory features used as inputs for the supervised machine learning algorithms are used as the independent variables in the linear model.

Fig. 5 presents the test set performance - mean squared errors (MSE), mean absolute error (MAE), and R-squared - of the three repair cost models-linear regression (LR), neural network (NN), and random forest (RF). Specifically, panel (a) plots the distribution of performance metrics generated by 100 random samples of the test set - 65\% without replacement - when all non-missing repair cost observations are included in model training and testing. Panel (b) presents the distribution of the same performance metrics when destroyed aircraft are excluded. The purpose of this exercise - removing destroyed aircraft during model training and testing - is to determine the extent to which rare, high-cost strike incidents influence the absolute and relative performance of the models.

Focusing first on panel (a) of Fig. 5, the model-specific distributions of the three different performance metrics show that random forest outperforms neural network and linear regression when predicting out-of-training-sample repair costs. This is evidenced by the density plots for random forest that are shifted left (right) for MSE and MAE (R-squared). In Table 3 we present the means and standard deviations that characterize these distributions (Panel A). Employing a simple $t$-test of means shows that both random forest and neural network provide a statistically significant improvement over linear regression ( $p<0.001$ for MSE, MAE, and R-squared), with random forest providing a statistically significant improvement over neural network ( $p<0.001$ for MSE, MAE, and R-squared). Specifically, random forest provides a 6.2\% improvement in MSE over linear regression, as well as a 3.6\% improvement in MAE and a 7\% improvement in R-squared. Compared to neural network, random forest provides a 3.5\% improvement in MSE, a $2.0 \%$ improvement in MAE, and a 3.7\% improvement in R-squared. Again, all of these differences are significant at traditional levels of statistical confidence.

The performance of all three models improves when destroyed aircraft observations are excluded from model training and testing - shown in panel (b) of Fig. 5. However, the improvement in predictive performance is much larger for neural network and linear regression than for random forest (Panel A, Table 3). So much so for the neural network, in fact, that it marginally outperforms random forest. In particular, neural network provides a 1\% improvement in MSE ( $p=0.049)$ over random forest and a $0.8 \%$ improvement in R-squared ( $p=0.013$ ) - the difference in MAE generated by neural network and random forest when destroyed aircraft are excluded is statistically indistinguishable from zero $(p=0.700)$. Note that, while the neural network provides a slight improvement over random forest in this case, random forest provides a more substantial improvement over neural network when destroyed aircraft are included in model training and testing. 
Table 3

Test set accuracy.

\begin{tabular}{|c|c|c|c|}
\hline & $\begin{array}{l}\text { Mean square error } \\
\text { Mean (SD) }\end{array}$ & $\begin{array}{l}\text { Mean absolute error } \\
\text { Mean (SD) }\end{array}$ & $\begin{array}{l}\text { R-squared } \\
\text { Mean (SD) }\end{array}$ \\
\hline \multicolumn{4}{|c|}{ Panel A: repair costs } \\
\hline \multicolumn{4}{|c|}{ Destroyed aircraft included } \\
\hline LR & $2.812(0.098)$ & $1.290(0.023)$ & $0.471(0.015)$ \\
\hline NN & $2.732(0.094)$ & $1.270(0.023)$ & $0.486(0.016)$ \\
\hline $\mathrm{RF}$ & $2.637(0.088)$ & $1.244(0.022)$ & $0.504(0.015)$ \\
\hline \multicolumn{4}{|c|}{ Destroyed aircraft excluded } \\
\hline LR & $2.586(0.090)$ & $1.253(0.021)$ & $0.513(0.013)$ \\
\hline NN & $2.483(0.087)$ & $1.227(0.022)$ & $0.532(0.013)$ \\
\hline $\mathrm{RF}$ & $2.508(0.090)$ & $1.226(0.023)$ & $0.528(0.013)$ \\
\hline \multicolumn{4}{|c|}{ Panel B: other costs } \\
\hline \multicolumn{4}{|c|}{ Destroyed aircraft included } \\
\hline LR & $2.104(0.168)$ & $0.929(0.022)$ & $0.937(0.005)$ \\
\hline NN & $1.871(0.110)$ & $0.868(0.021)$ & $0.944(0.004)$ \\
\hline $\mathrm{RF}$ & $1.822(0.109)$ & $0.838(0.021)$ & $0.945(0.003)$ \\
\hline \multicolumn{4}{|c|}{ Destroyed aircraft excluded } \\
\hline LR & $1.724(0.074)$ & $0.884(0.020)$ & $0.949(0.002)$ \\
\hline $\mathrm{NN}$ & $1.714(0.070)$ & $0.860(0.019)$ & $0.950(0.002)$ \\
\hline $\mathrm{RF}$ & $1.626(0.066)$ & $0.806(0.019)$ & $0.952(0.002)$ \\
\hline
\end{tabular}

NOTE: The means and standard deviations displayed in the table above are generated by testing each tuned algorithm - linear regression (LR), neural network (NN), and random forest (RF) - on 100 random samples of the test set. Figs. 5 and 6 plot the complete distributions of these measures. Each of the 100 random samples was $65 \%$ of the respective test set $-[1026 \times 0.65]=667$ randomly selected observations per sample for repair costs and $[1172 \times 0.65]=762$ for other costs.

Moving to our models of other costs, Fig. 6 plots the distributions of the same three performance metrics generated by 100 random samples of the other cost test set- $65 \%$ without replacement. Similar to repair costs, panels (a) and (b) of Fig. 6 present the distribution of performance metrics when destroyed aircraft observations are included and excluded during model training and testing, respectively. Unlike model performance on out-of-training-sample repair costs, random forest outperforms both linear regression and neural network across all three metrics regardless of the presence, or absence, of destroyed aircraft observations when predicting out-of-training-sample other costs (Panel B, Table 3). In particular, random forest provides a 13.4\% improvement in MSE ( $p<0.001)$, a 9.8\% improvement in MAE $(p<0.001)$, and a 0.9\% improvement in R-squared ( $p<0.001)$ over linear regression when destroyed aircraft observations are included, with these differences falling to 5.7\% $(p<0.001)$, 8.8\% $(p<0.001)$, and $0.3 \%(p<0.001)$, respectively, when destroyed aircraft are removed. Compared to neural network, random forest supplies a $2.6 \%$ improvement in MSE ( $p=0.009)$, a 3.5\% improvement in MAE $(p<0.001)$, and a $0.1 \%$ improvement in R-squared $(p=0.013)$. Upon the removal of destroyed aircraft strike observations these performance differences grow to $5.1 \%(p<0.001), 6.3 \%(p<0.001)$, and $0.2 \%(p<0.001)$, respectively. Interestingly, the performance of neural network relative to random forest deteriorated upon the removal of destroyed aircraft observations.

From these performance metrics we draw a number of conclusions. First, each algorithm - LR, RF, and NN - is better at predicting out-of-training sample other costs compared to out-of-training sample repair costs. This is no surprise given the high variance of reported repair costs relative to other costs in the NWSD. Second, random forest, and to a lesser extent neural network, provides a statistically significant and nontrivial improvement over linear regression in nearly every instance. This corroborates previous research on the benefit of using machine learning techniques over more traditional econometric methods when confronted with the problem of prediction (Verlinden et al., 2008; Mullainathan and Spiess, 2017). The ability of machine learning algorithms to outperform linear regressions in the realm of prediction can be attributed to their ability locate and prioritize the most important relationships - the most important relationships with respect to predictive performance. For instance, a fully interacted linear regression model and a regression tree that is built on the same set of explanatory features are generally equivalent when the explanatory features are categorical-i.e. conditional means at the intersection of explanatory feature characteristics. However, knowing which explanatory features and interactions that are most valuable with respect to prediction are often unknown to the researcher a priori. The random forest and neural network machine learning algorithms, along with others, are able to determine which relationships are most important and limit the extent to which restrictive structure is imposed on the data by the researcher.

Third, with respect to the prediction of out-of-training-sample repair costs, random forest is better able to handle the presence of rare, high-cost incidents. This is evidenced by the fact that random forest outperforms neural network and linear regression when destroyed aircraft observations are included in model training and testing, while the relative performance of neural network and linear regression improves upon the exclusion of these observations. With respect to the prediction of out-of-training-sample other costs, random forest outperforms neural network and linear regression when destroyed aircraft observations are both included and excluded in model training and testing. Taking all of these results together, we select the random forest models for our final imputation of both missing repair and other costs in the NWSD. 
(a) "Destroyed" aircraft included

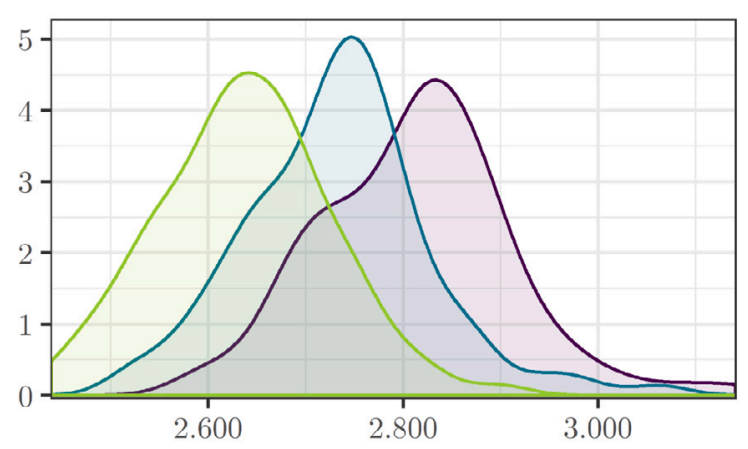

Mean Square Error



Mean Absolute Error

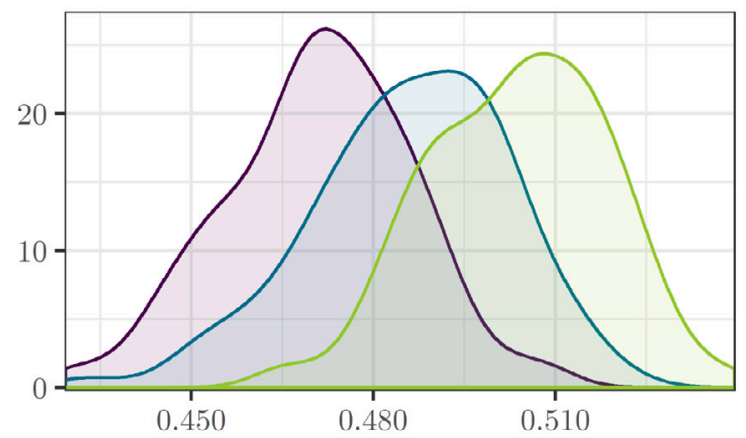

R-Squared (b) "Destroyed" aircraft excluded

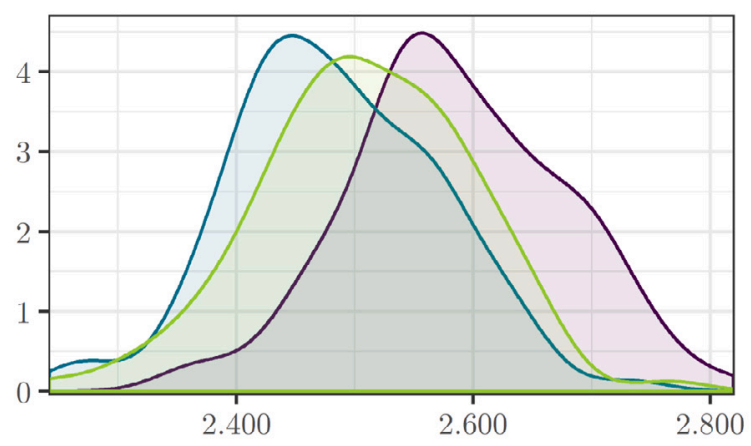

Mean Square Error

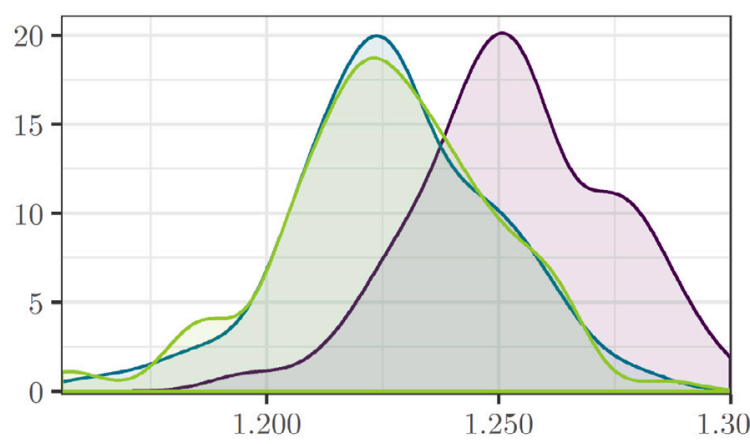

Mean Absolute Error

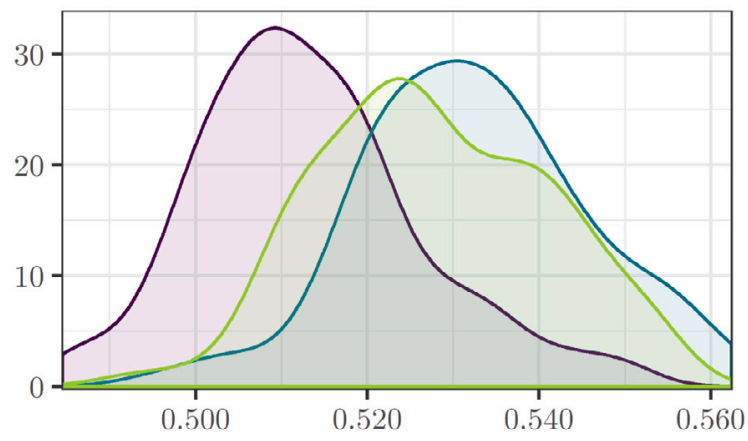

R-Squared

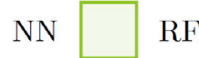

Fig. 5. Model performance on repair cost test set. NOTE: The distribution accuracy measures shown in the density plots above were generated by testing each tuned algorithm-linear regression (LR), neural network (NN), and random forest (RF)—on 100 random samples of the test set. Each random sample was $65 \%$ of the test set $(N=667)$. Panel (a) shows the distribution of test set accuracy measures when destroyed aircraft observations are included in model training and testing while panel (b) shows the distribution of accuracy measures when these observations are excluded. 
(a) "Destroyed" aircraft included

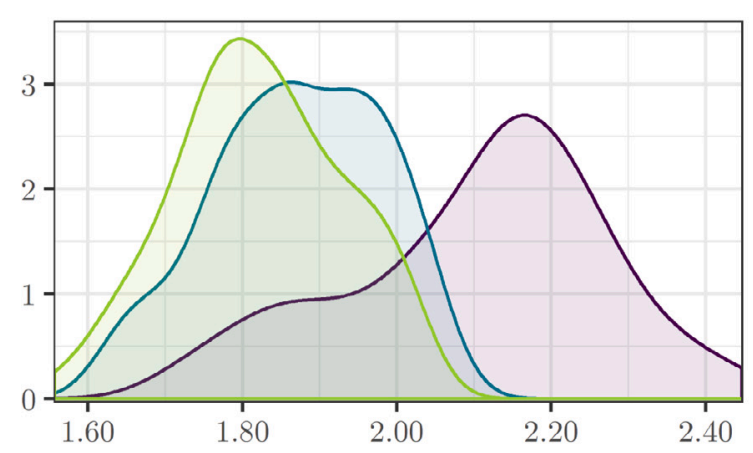

Mean Square Error

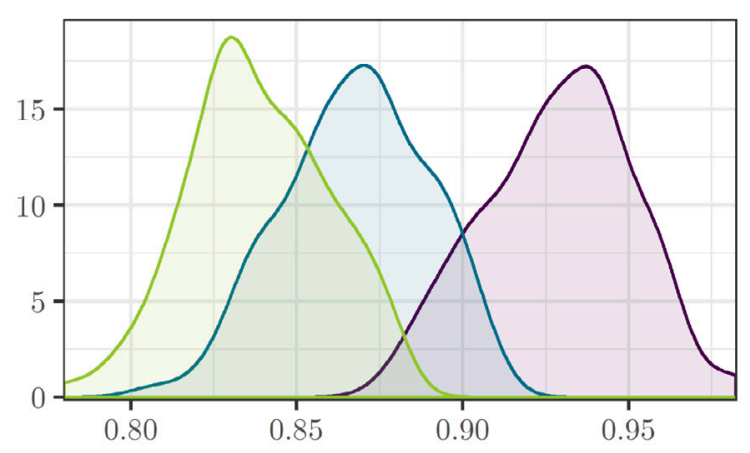

Mean Absolute Error

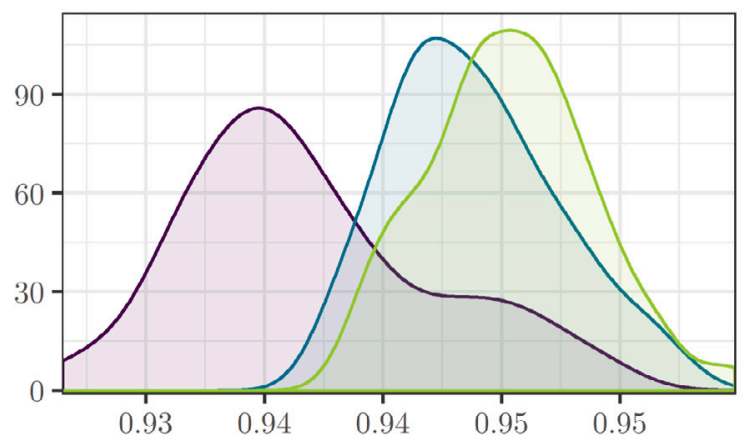

R-Squared (b) "Destroyed" aircraft excluded

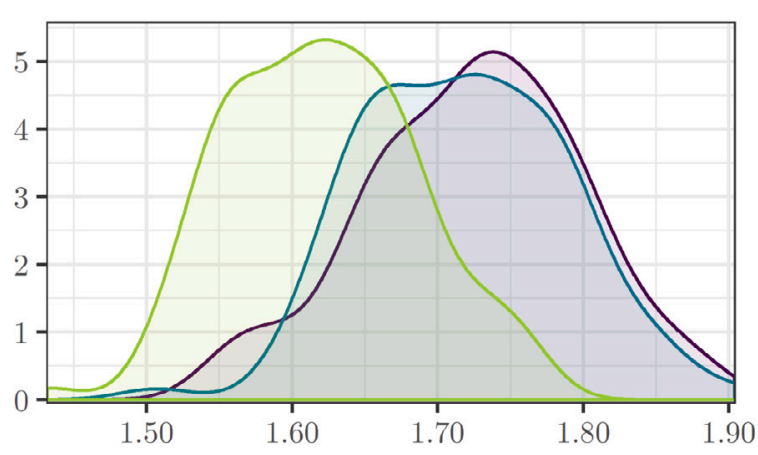

Mean Square Error

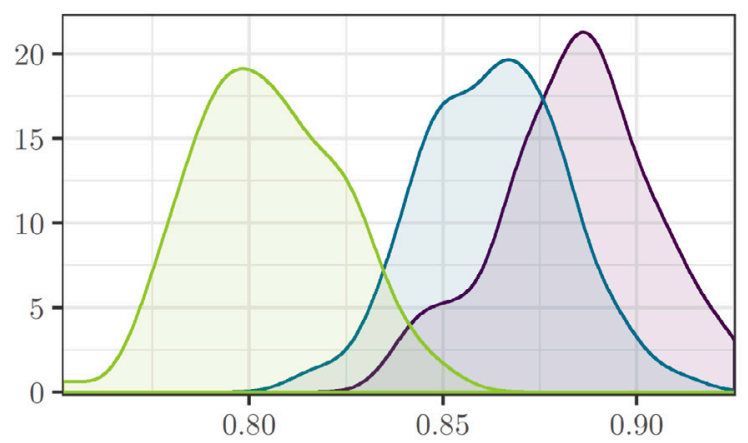

Mean Absolute Error

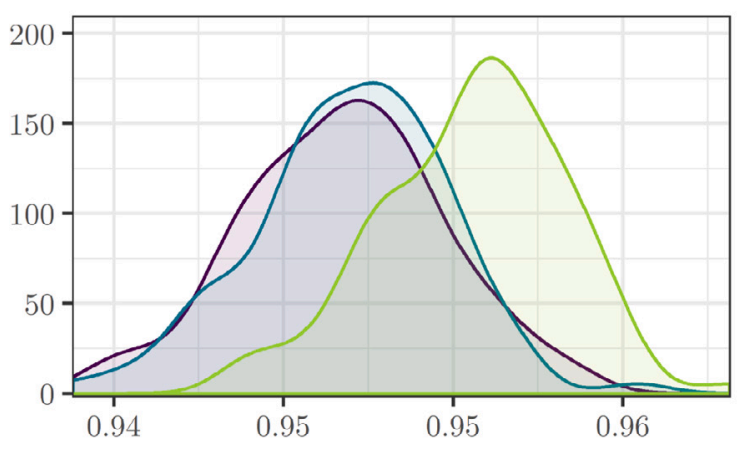

R-Squared

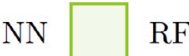

Fig. 6. Model performance on other costs test set. The distribution accuracy measures shown in the density plots above were generated by testing each tuned algorithm-linear regression (LR), neural network (NN), and random forest (RF)—on 100 random samples of the test set. Each random sample was $65 \%$ of the test set $(N=762)$. Panel (a) shows the distribution of test set accuracy measures when destroyed aircraft observations are included in model training and testing while panel (b) shows the distribution of accuracy measures when these observations are excluded. 


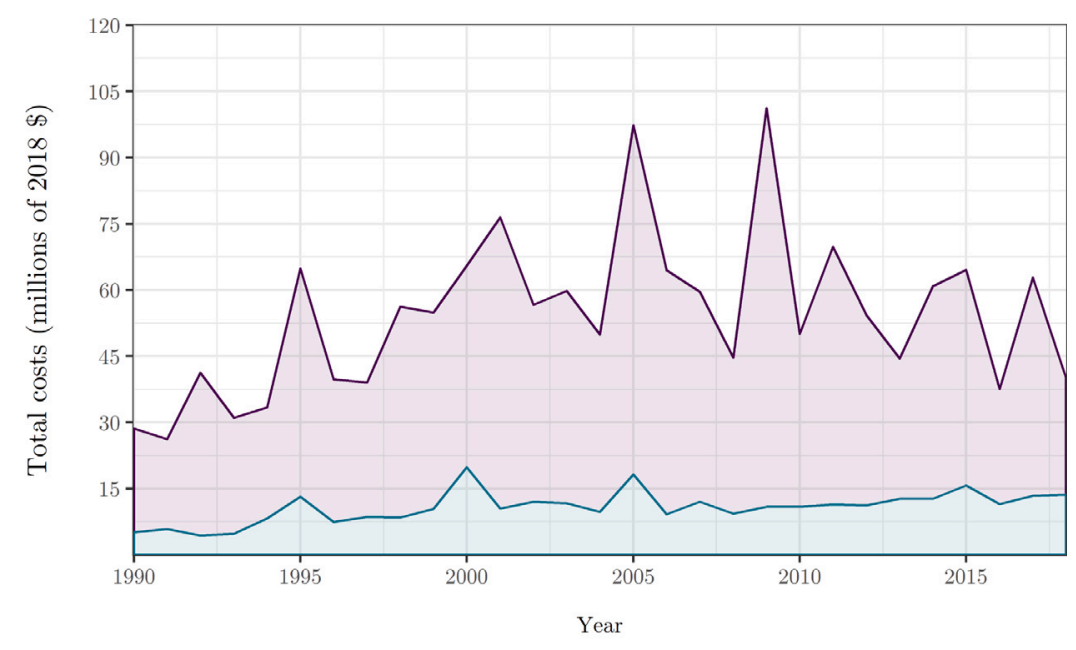

Repair costs

Other costs

Fig. 7. Lower bound total cost estimates, 1990-2018. NOTE: This figure plots the yearly lower bound machine learning estimates for other (blue) and repair (purple) costs over the period 1990-2018 measured in 2018 \$. The cost measures are stacked so that the uppermost line reflects the trend in total costs over the period. (For interpretation of the references to color in this figure legend, the reader is referred to the web version of this article.)

\subsection{Lower bound estimates of total costs}

Before imputing missing costs in the NWSD, the selected Random Forest repair and other cost models are retrained on all nonmissing cost observations-5,129 repair cost observations and 5,860 other cost observations. Final model performance on the 5,129 non-missing log repair cost observations is: $\mathrm{MSE}=1.47$, MAE $=0.94$, and R-squared $=0.73$. Of all the features included in our final repair cost model, the most important in predicting repair costs were: damage type (e.g., destroyed, substantial, minor, or uncertain), engine type (e.g., reciprocating engine (piston), turbofan, turbojet, etc.), animal size (e.g., small, medium, or large), whether the animal was ingested into the engine, whether the engine or radome was struck and/or damage, and aircraft mass. The relative importance of these variables is intuitive. For example. we would expect that the severity of damage would be a strong predictor of repair costs. Similarly, engine type along with engine damage should also be strong predictors of repair costs.

For our final model of other costs, performance on the 5,860 non-missing log other cost observations is: MSE $=1.02$, MAE $=0.62$, and R-squared $=0.97$. Of all the features included in our final other cost model, the most important in predicting other costs were: whether the strike resulted in damage or not, damage type (e.g., destroyed, substantial, minor, or uncertain), the number of wildlife seen and/or struck (e.g., 1, 2-10, or 11+), cloud cover (e.g., no clouds, some clouds, or overcast), whether the animal was ingested into the engine, and whether the pilot was warned or not. Similar to our repair cost model, damage and damage type are important predictors of other costs, as might be expected. Interestingly, however, the number of wildlife seen, cloud cover, and whether or not the pilot was warned about wildlife are also relatively important predictors of other costs in the NWSD.

Using these fully-trained random forest models, we impute missing costs in the NWSD. Then, after transforming the predicted log measures into dollar values (2018 \$), reported costs are combined with imputed costs to generate the yearly lower bound estimates of total costs (Fig. 7). It is important here that we emphasize our estimates as reflecting a lower bound of total costs that accrue to the US civil aviation industry, due to the previously mentioned issues of underreporting and unobserved external costs that arise from the delays imposed on passengers and the transport of goods and services. We decompose our estimates into repair (purple area) and other costs (blue area) which are stacked so that the uppermost line in Fig. 7 reflects the trend in total costs over the period. Repair costs comprise a majority of the costs that accrue to the US civil aviation industry as a result of aircraft-wildlife collisions, with our estimates indicating that the average yearly share of total costs coming from repairs is roughly 79.5 percent over the 1990-2018 period. Minimum average yearly total costs over the 1990-2018 period are estimated at \$54.3 million and \$61.1 million over the 2000-2018 period-see Appendix Table A.1.

Fig. 8 - which plots the data presented in Appendix Table A.1 - compares our yearly lower bound estimates to (1) actual reported total costs in the NWSD and (2) the estimates of Dolbeer et al. (2019). Our method of imputing missing costs in the NWSD increases average yearly reported costs by $80.1 \%$ - from $\$ 29.7$ million to $\$ 54.3$ million, specifically - for the $1990-2018$ period. This stands in stark contrast to the $529.6 \%$ increase - from $\$ 29.7$ million to $\$ 187$ million - produced by previous research. Fig. 8 clearly shows the drawbacks of the mean cost assignment method used to generate previous estimates. These estimates consistently sit well above our machine learning estimates, reflecting a consistent upward bias. Further, the estimates generated through mean 


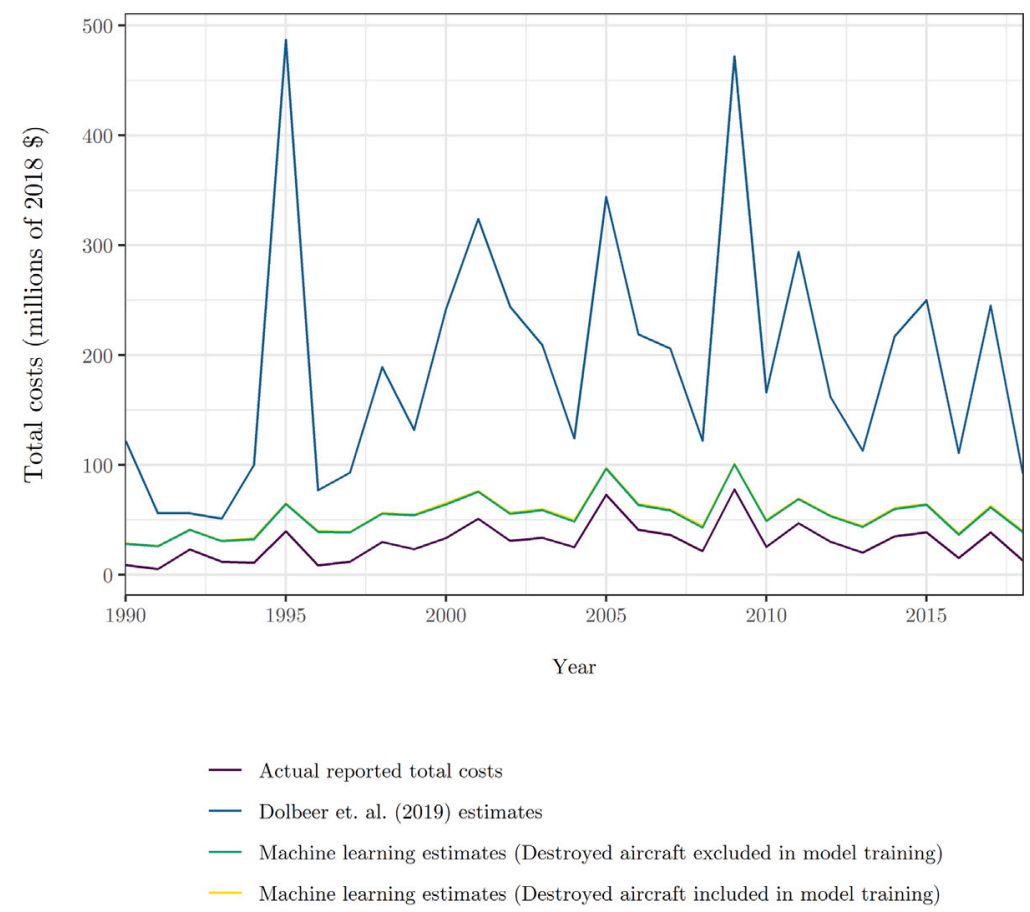

Fig. 8. Comparing lower bound cost estimates. NOTE: This figure compares the machine learning cost estimates to the yearly estimates provided by Dolbeer et al. (2019) in the FAA National Wildlife Strike Database Serial Report No. 25, as well as observed total costs reported to the NWSD. The Dolbeer et al. (2019) estimates were not recreated but were taken directly from Table 24 of the previously mentioned report.

cost assignment magnify the year-to-year variability of reported costs and produce relatively large spikes in years that contain rare, extremely high-cost incidents-e.g., 1995 and 2009.

In addition to our baseline machine learning estimates, where all non-missing cost observations included in model training, we generate additional estimates by excluding destroyed aircraft observations during final model training. This exercise provides a test of the sensitivity of our imputation method to the presence of rare strike incidents. We find that our method of cost imputation generates lower bound estimates that are effectively invariant to the presence of these low-probability, high-cost incidents (Fig. 8). Specifically, estimated minimum average yearly total costs over the 1990-2018 period is $\$ 54.3$ million when destroyed aircraft observations are included in final model training and \$53.5 million when destroyed aircraft observations are excluded ( Appendix Table A.1). As discussed in the background and motivation of this paper, the removal of the "Miracle on the Hudson" strike incident from the 2009 data reduced the mean cost assignment estimate in that year by $42 \%$. When we remove this observation, and others like it, from model training, our lower bound total cost estimate for 2009 falls by a mere $0.6 \%$. This difference is unobservable in Appendix Table A.1 due to rounding. Thus, we see our lower bound estimates of the economic burden of aircraft-wildlife collisions to the US aviation industry as providing a more accurate and robust alternative to previous cost estimates.

\section{Conclusion}

In this paper we exploit the presence of cost-relevant information provided for each strike report in the NWSD and employ emerging machine learning techniques to provide a more accurate lower bound estimate of monetary losses that accrue to the US civil aviation industry as a result of aircraft-wildlife collisions. Our results reflect, and contribute to, a growing consensus in empirical research that machine learning techniques are a more effective tool than traditional econometric methods when faced with the problem of missing information and prediction. In the context of costs reported to the NWSD, we find that random forest outperforms neural network in predicting repair and other costs through its ability to better handle the presence of rare, high-cost strike incidents that appear intermittently in the data. Employing the fully trained random forest models we impute missing costs in the NWSD and estimate that wildlife strikes generated a minimum of $\$ 54.3$ million in real annual monetary losses to the US civil aviation industry over the 1990-2018 period, as opposed to the $\$ 187$ million lower bound estimate provided by mean cost assignment methods. Then, testing the exclusion of destroyed aircraft strike observations, we find that our lower bound cost estimates are effectively invariant to the presence of rare, high-cost strike incidents.

A number of implications arise from our results. First, the methods employed by previous research have potentially overestimated the economic burden of aircraft-wildlife collisions to the US aviation industry. Even after taking into consideration the issue of underreporting, which may inflate our cost estimates by a factor of 2 or 3, our estimates still fall below those of previous researchers. 
Table A.1

A comparison of lower bound cost estimates (in millions of 2018 \$).

\begin{tabular}{|c|c|c|c|c|}
\hline Year & $\begin{array}{l}\text { Actual reported } \\
\text { costs }\end{array}$ & $\begin{array}{l}\text { Dolbeer et al. } \\
\text { (2019) estimates }\end{array}$ & ML estimates & $\begin{array}{l}\text { ML estimates } \\
\text { (Destroyed } \\
\text { aircraft } \\
\text { excluded) }\end{array}$ \\
\hline 1990 & 9 & 122 & 29 & 28 \\
\hline 1991 & 5 & 56 & 26 & 26 \\
\hline 1992 & 23 & 56 & 41 & 41 \\
\hline 1993 & 12 & 51 & 31 & 31 \\
\hline 1994 & 11 & 100 & 33 & 32 \\
\hline 1995 & 40 & 487 & 65 & 64 \\
\hline 1996 & 9 & 77 & 40 & 39 \\
\hline 1997 & 12 & 93 & 39 & 39 \\
\hline 1998 & 30 & 189 & 56 & 56 \\
\hline 1999 & 23 & 132 & 55 & 54 \\
\hline 2000 & 33 & 242 & 65 & 64 \\
\hline 2001 & 51 & 324 & 76 & 76 \\
\hline 2002 & 31 & 244 & 57 & 56 \\
\hline 2003 & 34 & 209 & 60 & 59 \\
\hline 2004 & 25 & 124 & 50 & 48 \\
\hline 2005 & 73 & 344 & 97 & 97 \\
\hline 2006 & 41 & 219 & 65 & 64 \\
\hline 2007 & 36 & 206 & 60 & 59 \\
\hline 2008 & 21 & 122 & 45 & 43 \\
\hline 2009 & 78 & 472 & 101 & 101 \\
\hline 2010 & 26 & 166 & 50 & 49 \\
\hline 2011 & 47 & 294 & 70 & 69 \\
\hline 2012 & 30 & 162 & 54 & 53 \\
\hline 2013 & 20 & 113 & 44 & 43 \\
\hline 2014 & 35 & 217 & 61 & 60 \\
\hline 2015 & 39 & 250 & 65 & 64 \\
\hline 2016 & 15 & 111 & 38 & 36 \\
\hline 2017 & 39 & 245 & 63 & 61 \\
\hline 2018 & 13 & 92 & 40 & 39 \\
\hline \multicolumn{5}{|l|}{ Average: } \\
\hline 1990-2018 & 29.7 & 187.0 & 54.3 & 53.5 \\
\hline 2000-2018 & 36.2 & & 61.1 & 60.1 \\
\hline
\end{tabular}

NOTE: This table compares the machine learning cost estimates to the yearly estimates provided by Dolbeer et al. (2019) in the FAA National Wildlife Strike Database Serial Report No. 25, as well as observed total costs reported to the NWSD. The (Dolbeer et al., 2019) estimates were not recreated but were taken directly from Table 24 of the previously mentioned report.

Further, it is likely that the most disruptive and severe strikes are more often reported to the NWSD so that unreported strikes are, on average, less costly than those which appear in the NWSD-i.e., an issue of selection in reporting. This suggests that previous estimates may actually be a reasonable upper, not lower, bound approximation of strike-related costs borne by the US civil aviation industry. However, it is important that we reiterate the failure of our estimates to capture external costs that stem from the delayed transport of passengers, as well as goods and services. Additionally, we do not account for the costs of morbidity and mortality in the rare instance that an aircraft-wildlife collision results in injury or fatality. Of the 237,305 total strike reports in the NWSD at the time of writing, $251(0.1 \%)$ indicate that the strike resulted in injury - a total of 328 persons injured - and $15(0.006 \%)$ indicate that the strike was fatal-a total of 35 fatalities. Further, we suspect that other costs reported to the NWSD, and, therefore, our estimates, may be underestimated given the difficulty, or even impossibility, of accounting for all of the indirect costs associated with a wildlife strike-especially if an individual is reporting a strike before potential future indirect costs have been realized. A serious account of the external costs that fall on all affected parties is an important area for future research.

Second, our improved estimates provide a first step in more accurately measuring the benefits of wildlife management at airports. In so far as wildlife management activities are effective in reducing the number and intensity of strike incidents that occur at a given airport, an accurate measure of the benefit of these activities requires that one has an accurate cost estimate for the strikes that would have occurred. Using our model, which makes extensive use of observed strike characteristics to predict costs in the NWSD, we can estimate the value of avoided strikes even for airports that have no previously recorded cost information in the NWSD. Moreover, our method of cost imputation allows us to calculate the cost of a typical strike at airports which never appear in the NWSD, given that we have sufficient information on the explanatory features included in our model-e.g., the typical type of aircraft at the airport, the typical mass of aircraft, the typical size of the wildlife that pose a strike risk at the airport, and so on.

Lastly, and building on the first two implications, our cost estimates can provide a measure of relative risk at airports beyond relative measures of strikes, disruptive strikes, and damaging strikes. For instance, though two airports may have similar levels of risk as measured by disruptive and damaging strikes per movement, our improved cost estimates may determine that the cost per 
damaging strike at one airport is relatively large compared to others. Thus, our research can assist in the efficient allocation of wildlife management resources.

\section{CRediT authorship contribution statement}

Levi Altringer: Conceptualization, Methodology, Data curation, Investigation, Formal analysis, Writing - original draft. Jordan Navin: Conceptualization, Methodology, Data curation, Investigation, Formal analysis, Writing - review \& editing. Michael J. Begier: Funding acquisition. Stephanie A. Shwiff: Writing - review \& editing, Supervision, Project administration, Funding acquisition. Aaron Anderson: Conceptualization, Methodology, Writing - review \& editing, Supervision.

\section{Declaration of competing interest}

The authors declare that they have no known competing financial interests or personal relationships that could have appeared to influence the work reported in this paper.

\section{Appendix}

See Table A.1.

\section{References}

Abdelwahab, H.T., Abdel-Aty, M.A., 2001. Development of artificial neural network models to predict driver injury severity in traffic accidents at signalized intersections. Transp. Res. Rec. 1746 (1), 6-13. http://dx.doi.org/10.3141/1746-02.

Anderson, A., Carpenter, D.S., Begier, M.J., Blackwell, B.F., DeVault, T.L., Shwiff, S.A., 2015. Modeling the cost of bird strikes to US civil aircraft. Transp. Res. D 38, 49-58. http://dx.doi.org/10.1016/j.trd.2015.04.027.

Arnold, T.B., 2017. KerasR: R interface to the keras deep learning library. J. Open Sour. Softw. 2 (14), 296. http://dx.doi.org/10.21105/joss.00296.

Chang, L.-Y., 2005. Analysis of freeway accident frequencies: negative binomial regression versus artificial neural network. Saf. Sci. 43 (8), 541-557. http://dx.doi.org/10.1016/j.ssci.2005.04.004.

Cleary, E.C., Dolbeer, R.A., 2005. Wildlife Hazard Management at Airports: A Manual for Airport Personnel. USDA National Wildlife Research Center - Staff Publications, URL https://digitalcommons.unl.edu/icwdm_usdanwrc/133?utm_source=digitalcommons.unl.edu\%2Ficwdm_usdanwrc\%2F133\&utm_medium=PDF\&utm_ campaign=PDFCoverPages.

DeVault, T.L., Belant, J.L., Blackwell, B.F., Seamans, T.W., 2011. Interspecific variation in wildlife hazards to aircraft: Implications for airport wildlife management. Wildl. Soc. Bull. 35 (4), 394-402. http://dx.doi.org/10.1002/wsb.75.

Dolbeer, R.A., 2015. Trends in Reporting of Wildlife Strikes with Civil Aircraft and in Identification of Species Struck Under a Primarily Voluntary Reporting System, 1990-2013. Technical Report, Federal Aviation Administraiton, Special Report, URL https:/digitalcommons.unl.edu/zoonoticspub/188/.

Dolbeer, R.A., Begier, M.J., Miller, P.R., Weller, J.R., Anderson, A.L., 2019. Wildlife Strikes to Civil Aircraft in the United States 1990-2018. Technical Report, Federal Aviation Administration, National Wildlife Strike Database, Serial Report Number 25, URL https://www.faa.gov/airports/airport_safety/wildlife/ media/Wildlife-Strike-Report-1990-2018.pdf.

FAA, 2020. National Wildlife Strike Database. Online Database. Federal Aviation Administration, URL https://wildlife.faa.gov/home.

Jiang, Y., Cukic, B., Menzies, T., 2008. Does transformation help. Defects (2008b) URL http://unbox.org/wisp/var/issta/multipleLearners.pdf.

Liaw, A., Wiener, M., et al., 2002. Classification and regression by randomforest. R News 2 (3), 18-22, URL https://www.researchgate.net/profile/ Andy_Liaw/publication/228451484_Classification_and_Regression_by_RandomForest/links/53fb24cc0cf20a45497047ab/Classification-and-Regression-byRandomForest.pdf.

Mannering, F.L., Bhat, C.R., 2014. Analytic methods in accident research: Methodological frontier and future directions. Anal. Methods Accid. Res. 1, 1-22. http://dx.doi.org/10.1016/j.amar.2013.09.001.

Mannering, F., Bhat, C.R., Shankar, V., Abdel-Aty, M., 2020. Big data, traditional data and the tradeoffs between prediction and causality in highway-safety analysis. Anal. Methods Accid. Res. 25, 100113. http://dx.doi.org/10.1016/j.amar.2020.100113.

Metz, I.C., Ellerbroek, J., Mühlhausen, T., Kügler, D., Hoekstra, J.M., 2020. The bird strike challenge. Aerospace 7 (3), 26. http://dx.doi.org/10.3390/ aerospace7030026.

Moisen, G., 2008. Classification and regression trees. In: Fath, S.E.J.B.D. (Ed.), Encyclopedia of Ecology, Vol. 1, first ed. Elsevier, pp. 582-588, URL https://www.fs.fed.us/rm/pubs_other/rmrs_2008_moisen_g001.pdf.

Mullainathan, S., Spiess, J., 2017. Machine learning: an applied econometric approach. J. Econ. Perspect. 31 (2), 87-106. http://dx.doi.org/10.1257/jep.31.2.87.

Navin, J., Weiler, S., Anderson, A., 2020. Wildlife strike cost revelation in the US domestic airline industry. Transp. Res. D 78, 102204 . http://dx.doi.org/10. 1016/j.trd.2019.102204.

Ramachandran, P., Zoph, B., Le, Q.V., 2017. Searching for activation functions. arXiv preprint arXiv:1710.05941. URL https://arxiv.org/abs/1710.05941.

Raschka, S., 2018. Model evaluation, model selection, and algorithm selection in machine learning. arXiv preprint arXiv:1811.12808. URL https://arxiv.org/abs/ 1811.12808.

Riviere, C., Lauret, P., Ramsamy, J.M., Page, Y., 2006. A Bayesian neural network approach to estimating the energy equivalent speed. Accid. Anal. Prev. 38 (2), 248-259. http://dx.doi.org/10.1016/j.aap.2005.08.008.

Roca-González, J.-L., Vera-Lopez, J.-A., Rodriguez-Bermudez, G., 2020. Organisational and costing aspects to prevent wildlife strikes on airports: A case study of Spanish airport security managers. Saf. Sci. 122, 104520.

Tang, J., Zheng, L., Han, C., Yin, W., Zhang, Y., Zou, Y., Huang, H., 2020. Statistical and machine-learning methods for clearance time prediction of road incidents: A methodology review. Anal. Methods Accid. Res. 27, 100123. http://dx.doi.org/10.1016/j.amar.2020.100123.

Verlinden, B., Duflou, J., Collin, P., Cattrysse, D., 2008. Cost estimation for sheet metal parts using multiple regression and artificial neural networks: A case study. Int. J. Prod. Econ. 111 (2), 484-492. http://dx.doi.org/10.1016/j.ijpe.2007.02.004.

Xie, Y., Lord, D., Zhang, Y., 2007. Predicting motor vehicle collisions using Bayesian neural network models: An empirical analysis. Accid. Anal. Prev. 39 (5), 922-933. http://dx.doi.org/10.1016/j.aap.2006.12.014. 